\title{
Appropriate Moderated Regression and Inappropriate Research Strategy: A Demonstration of Information Loss Due to Scale Coarseness
}

\author{
Craig J. Russell \\ Rutgers University \\ Jeffrey K. Pinto \\ University of Maine \\ Philip Bobko \\ Rutgers University
}

\begin{abstract}
Paunonen and Jackson (1988) demonstrated that stepwise moderated regression provides a test of interaction effects that protects the nominal Type I error rate. However, the stepwise procedure has also been characterized as failing to detect interaction effects in empirical studies. This issue has led to questions regarding the method's statistical power (Bobko, 1986; Zedeck, 1971) in applied research. It is demonstrated that, because of a research strategy frequently used in empirical investigations, the probability of Type II error in detecting a true interaction effect is unknown. Specifically, the number of scale steps used in measuring the dependent variable is shown to result in a form of systematic error that can spuriously increase or decrease the expected effect size of the interaction. The problem is also discussed in the context of testing more complex models. Recommendations for eliminating this problem in future research designs are provided. Index terms: information loss, interaction effects, Likert scales, moderated regression, response transformation.
\end{abstract}

Stepwise moderated regression analysis was first described by Saunders $(1955,1956)$ as a statistical tool for assessing moderator effects at an individual level of analysis. Shortly thereafter, Chow (1960) described an identical procedure for assessing interaction effects at a macro level of organizational

APPLIED PSYCHOLOGICAL MEASUREMENT

Vol. 15, No. 3, September 1991, pp. 257-266

(C) Copyright 1991 Applied Psychological Measurement Inc. 0146-6216/91/030257-10\$1.75 research. Since that time, the infrequency in the applied psychological literature of findings supportive of moderator effects has fueled concerns over the power of the procedure (Zedeck, 1971). More recently, Bobko (1986) and Venkatraman (1989) have reported specific concerns regarding the correspondence or "fit" between an interactive conceptual framework and statistical procedures used to evaluate the framework.

The difficulty in detecting interactions has often been attributed to multicollinearity among predictor variables, particularly between a multiplicative interaction and its respective component main effects (Drazin \& Van de Ven, 1985; Sockloff, 1976a, 1976b). Unfortunately, recent attempts to statistically control for multicollinearity effects (e.g., Morris, Sherman, \& Mansfield, 1986) have not proved fruitful (Cronbach, 1987). Although Paunonen and Jackson (1988) demonstrated that stepwise moderated regression is not susceptible to inflation of Type I error in the presence of multicollinearity, Cronbach (1987) has recently called for more sensitive research strategies for the detection of interaction effects.

Because the statistical procedure is sound, what alternate research strategies should be pursued? At best, an investigator is confronted with a well-formulated theory that dictates the strategy of choice. For example, Bobko (1986) demon- 
strated a research strategy of specific cell contrasts for theories hypothesizing particular ordinal interactions. Unfortunately, as noted by Cronbach (1987) and Venkatraman (1989), applied researchers are frequently faced with models that are weakly specified.

Yet another strategy focuses on the reduction of measurement error (Schwab, 1980). Busemeyer and Jones (1983) demonstrated how measurement error influences the power of moderated regression (see their Equation 9). Specifically, suppose a regression model attempts to predict a criterion $(Y)$ from two independent variables $\left(X_{1}, X_{2}\right)$ and their interaction. Busemeyer and Jones (1983) demonstrated that measurement error in the independent variables $X_{1}$ and $X_{2}$ reduces the expected effect size of moderated regression (i.e., the difference in the squared multiple correlation, $R^{2}$, between the multiplicative and additive models) when a true multiplicative relationship exists between $X_{1}$ and $X_{2}$ and the dependent variable $Y$. Using instruments with known reliabilities and Busemeyer and Jones' formula, investigators can determine the expected effect size if a true multiplicative relationship exists and hence identify sample size needed to yield adequate statistical power.

Cohen (1983) and Peters and Van Voorhis (1940) demonstrated the impact of information loss in the dependent variable on simple Pearson product-moment correlations. This information loss may occur when a continuous dependent variable is reduced to a small set of categories by the investigator in a known way. Cox (1950) demonstrated how to minimize the loss of information by selecting optimally-sized groups of observations taken from a continuous distribution. All prior work in this area has focused on information loss that occurs in some known fashion. However, no study has examined the consequence of persons responding to a discrete scale when, in fact, the underlying construct is continuous. This paper demonstrates that the number of options on the dependent scale will be a source of unknown systematic error in moderated regression and, in turn, will spuriously increase or decrease underlying statistical power.

\section{Systematic Error and Information Loss}

In applied research, most constructs of interest are continuous. The actual number of options available on response scales is often discrete. That is, $X_{1}, X_{2}$, and $Y$ are often measured on 1- to 5-point Likert scales (e.g., Stahl \& Harrell, 1981; Russell, 1985). Furthermore, note that if the three variables are all measured on 5-point Likert scales, then the empirical interaction term $X_{1} \times X_{2}$ is arithmetically defined by a $1-$ to 25 -point scale. Thus, the measurement of $Y$ ( 1 to 5) does not necessarily map, in any straightforward way, onto the 25 -point interaction term from the regression analysis. Information is lost when a "coarse" response scale is used to represent a near continuous or "fine" $Y$ variable. As demonstrated below, this phenomenon has direct implications for systematic contributions to error variation in the Likert response format.

The original Likert method of scale construction involved the operationalization of continuous psychological constructs by summing responses to questions of opinion (Likert, 1932). Likert's response scales contained five scale steps (strongly agree, agree, undecided, disagree, and strongly disagree). Likert and others demonstrated that this procedure resulted in low levels of random error variation (i.e., high reliability) in resultant scale scores (Edwards \& Kenney, 1946; Likert, 1932; Rasmussen, 1989). Furthermore, Cicchetti, Showalter, and Tyrer (1985) and Jenkins and Taber (1977), using monte carlo studies, demonstrated that no substantial reduction in random error occurs when the number of scale steps exceeds a range of five to seven points.

However, systematic error may be introduced when respondents are faced with a fine, true underlying response and a coarse, overt 5-point response scale. The systematic error occurs when information from the fine/true response is lost as it is placed on a coarse 5-point response scale. Although Cohen (1983) and Peters and Van Voorhis (1940) demonstrated the impact on sim- 
ple linear regression analyses when the investigator decides how to transform a continuous $Y$ variable into categories in some known way, the impact of requiring respondents to perform the transformation in some unknown way has not been considered. It is hypothesized that the increased range of potential values characterizing a "true" response in interactive regression will lead to even greater information loss and larger opportunity for systematic error than in simple linear regression. Because people respond to only one question at a time, dependent scale scores derived by summing responses to multiple Likerttype items will also suffer from this information loss.

For example, in a test of a multiplicative expectancy theory model, Stahl and Harrell (1981) used a within-persons design with 11 levels of valence, three levels of expectancy, and an 11-point dependent response scale. If respondents used a multiplicative function of expectancy and valence to arrive at estimates of motivational force, they were faced with placing a 33-point $(3 \times 11)$ latent response space onto an overt 11-point dependent scale. Interestingly, the findings of Stahl and Harrell failed to support a multiplicative expectancy model for the majority of respondents.

In contrast, Arnold (1981) used a withinpersons design with five levels of each independent variable (expectancy and valence) and 150 levels of the dependent variable (motivational force). A multiplicative expectancy theory model (force $=$ expectancy $\times$ valence) would require 25 points in the dependent response space. Using a 150-point response scale, Arnold certainly gave respondents sufficient options on $Y$ to portray their "true" response, regardless of whether a multiplicative or additive model was correct. In contrast to Stahl and Harrell (1981), Arnold found substantial evidence supporting the multiplicative formulation of expectancy theory. These conflicting findings may be related to the investigators' choice of response scales. The presence of a true interaction effect can create more latent responses than actual response op- tions available. When respondents are faced with a scale on the dependent variable that does not have a sufficient number of response options, information loss is unavoidable.

The purpose of the analyses presented below was to demonstrate how information loss caused by the overt response scale has an unknown influence on effect sizes found in moderated regression analysis. For purposes of explication, two examples of hypothetical regression data were generated in which the dependent scale $(Y)$ did not have a sufficient number of options to reflect either a multiplicative effect or an additive effect. An additive example demonstrates one way information might be lost when two variables are additively combined.

\section{Multiplicative Example}

\section{The Data}

Three sets of dependent responses were generated for purposes of illustration. Dependent scales $Y_{1}, Y_{2}$, and $Y_{3}$ were generated from the same array of $X_{1}$ and $X_{2}$ observations. Each independent variable was characterized by a 5 -point scale $(1,2,3,4,5)$ in a fixed effects two-way ANOVA design (each level of $X_{1}$ and $X_{2}$ occurred with equal frequency). Thus, $X_{1}$ and $X_{2}$ each had a mean of 3 and standard deviation of 1.41 (each value of $X_{1}$ was paired with every value of $X_{2}$ so that $r_{1,2}=0.0$ ).

The dependent scale $\left(Y_{1}\right)$ was created simply by multiplying $X_{1}$ and $X_{2}\left(Y_{1}=X_{1} \times X_{2}\right)$. The resultant response range was from 1 to 25 and represented the "true score" response expected from a respondent who used a multiplicative combination of $X_{1}$ and $X_{2}$, who responded to the dependent variable response scale $\left(Y_{1}\right)$ with no random measurement error, and who was given a dependent variable response scale that permitted all responses to be made without any information loss.

Information loss in the second dependent scale $\left(Y_{2}\right)$ was created in one step by multiplying $X_{1}$ and $X_{2}$ and then recoding the data into five equal intervals. Specifically, all products of $X_{1}$ and $X_{2}$ be- 
tween 1 and 5 were assumed to elicit a mean overt response on the $Y_{2}$ scale of 1 , original $Y_{1}$ values between 6 and 10 were assumed to elicit a mean overt $Y_{2}$ response of 2, and so forth. This was meant to represent what might happen when a 5-point dependent variable response scale is used to portray a 25 -point latent response space. With $Y_{1}$ representing the respondent's "true" original response, $Y_{2}$ represented the response expected from a respondent who used a multiplicative combination of $X_{1}$ and $X_{2}$, who responded to the dependent variable response scale $\left(Y_{2}\right)$ with no random measurement error, and who was given a coarse dependent response scale that caused information to be lost from the "true" $Y_{1}$ response.

Information loss in the third dependent scale $\left(Y_{3}\right)$ was created in two steps by taking the square root of the product of $X_{1}$ and $X_{2}$ and rounding to the nearest integer. Although it seems unlikely that respondents can perform square root functions "in their heads," there is strong evidence from laboratory investigations of psychophysical scaling that respondents do perform the mathematical equivalent of this procedure (Anderson, 1982; Birnbaum \& Veit, 1974). There is no reason to believe that the $Y_{2}$ or $Y_{3}$ transformation is more appropriate than the other. However, information must be eliminated somehow if the "true" response is to be placed on the five-point scale, and $Y_{2}$ and $Y_{3}$ are both viable examples of how information loss might occur. Table 1 contains all possible combinations of $X_{1}$ and $X_{2}$ variables with their associated $Y_{1}, Y_{2}$, and $Y_{3}$ responses.

\section{Analyses and Results}

Stepwise moderated regression analysis was performed for each of the dependent variables $Y_{1}, Y_{2}$, and $Y_{3}$. The effect size of interest was $R_{\text {mult }}^{2}-R_{\text {add }}^{2}$ from the following equations (Cohen \& Cohen, 1983):

For $R_{\text {add }}^{2}$

$Y=\beta_{0}+\beta_{1} X_{1}+\beta_{2} X_{2}+\varepsilon$.

For $R_{\text {mult }}^{2}$

$$
Y=\beta_{0}^{*}+\beta_{1}^{*} X_{1}+\beta_{2}^{*} X_{2}+\beta_{3}^{*} X_{1} X_{2}+\varepsilon .
$$

Table 1

Complete Set of Independent Variables and Dependent Variables for Multiplicative Example

\begin{tabular}{rrrrr}
\hline \hline$X_{1}$ & $X_{2}$ & $Y_{1}$ & $Y_{2}$ & $Y_{3}$ \\
\hline 1 & 1 & 1 & 1 & 1 \\
1 & 2 & 2 & 1 & 1 \\
1 & 3 & 3 & 1 & 2 \\
1 & 4 & 4 & 1 & 2 \\
1 & 5 & 5 & 1 & 2 \\
2 & 1 & 2 & 1 & 1 \\
2 & 2 & 4 & 1 & 2 \\
2 & 3 & 6 & 2 & 2 \\
2 & 4 & 8 & 2 & 3 \\
2 & 5 & 10 & 2 & 3 \\
3 & 1 & 3 & 1 & 2 \\
3 & 2 & 6 & 2 & 2 \\
3 & 3 & 9 & 2 & 3 \\
3 & 4 & 12 & 3 & 3 \\
3 & 5 & 15 & 3 & 4 \\
4 & 1 & 4 & 1 & 2 \\
4 & 2 & 8 & 2 & 3 \\
4 & 3 & 12 & 3 & 3 \\
4 & 4 & 16 & 4 & 4 \\
4 & 5 & 20 & 4 & 4 \\
5 & 1 & 5 & 1 & 2 \\
5 & 2 & 10 & 2 & 3 \\
5 & 3 & 15 & 3 & 4 \\
5 & 4 & 20 & 4 & 4 \\
5 & 5 & 25 & 5 & 5 \\
\hline & & & &
\end{tabular}

These effect sizes are reported in Table 2. First, note that $R_{\text {mult }}^{2}=1.00$ for $Y_{1}$, because $Y_{1}$ was constructed as the product of $X_{1}$ and $X_{2}$ with no random measurement error. Further, when there was no information loss due to limited response options, $R_{\text {add }}^{2}=.90$ and the incremental effect for the interaction term is $R_{\text {mul }}^{2}-R_{\text {add }}^{2}=.10$. In contrast, if the 5-point dependent response scale caused information loss due to rounding latent responses within equal-sized intervals (i.e., $Y_{2}$ ), the additive effect decreased and the expected

Table 2

Moderated Regression Effect Sizes

\begin{tabular}{lrcc}
\hline \hline Dependent & & & \\
Variable & $R_{\text {mult }}^{2}$ & $R_{\text {add }}^{2}$ & $R_{\text {muli }}^{2}-R_{\text {add }}^{2}$ \\
\hline$Y_{1}$ & 1.000 & .900 & .100 \\
$Y_{2}$ & .961 & .781 & .180 \\
$Y_{3}$ & .934 & .911 & .023 \\
\hline
\end{tabular}


effect size for the interaction increased to .180 . When the 5-point dependent response scale caused information loss through rounding the square root of the latent response to the nearest integer (i.e., $Y_{3}$ ), the expected interaction effect size decreased to .023 .

It should be noted that the above univariate distributions of $X_{1}$ and $X_{2}$ were uniform. When the procedures were replicated under conditions of normally distributed and/or correlated independent variables, the pattern of results was the same.

It is of interest to note that relative to $Y_{1}$, the $R_{\text {add }}^{2}$ for $Y_{2}$ was substantially lower and the $R_{\text {add }}^{2}$ for $Y_{3}$ was slightly higher. $R_{\text {mult }}^{2}$ decreased for both $Y_{2}$ and $Y_{3}$. It is the differential size of these changes that contributed to inflation or deflation of $R_{\text {mult }}^{2}-R_{\text {add }}^{2}$ for the $Y_{2}$ and $Y_{3}$ transformations. There is no apparent theoretical explanation that might account for the substantial drop in $R_{\text {add }}^{2}$ for $Y_{2}$. However, Table 1 suggests that the $Y_{2}$ transformation resulted in a disproportionate amount of the "systematic" error occurring in the lower range of values, resulting in moderate range restriction (there were more 1s for $Y_{2}$ than for $Y_{3}$ ). Although both the $Y_{2}$ and $Y_{3}$ transformations diminished a strong rank ordering among the 25 possible "true" responses, the effect of the resultant "ties" or grouping of $Y_{1}$ values into the "1" category of $Y_{2}$ had a stronger attenuation effect on $R_{\text {add }}^{2}$ than was the case for $Y_{3}$.

\section{An Additive Example}

\section{The Data}

Two sets of dependent responses were generated. Dependent scales $Y_{1}$ and $Y_{2}$ were generated from the previous array of $X_{1}$ and $X_{2}$ observations. As in the multiplicative example, each independent variable was characterized by a 5-point scale with each value observed with equal frequency.

The first dependent scale $\left(Y_{1}\right)$ was created by simply adding $X_{1}$ and $X_{2}$ (again, under the assumption of no random measurement error). The resultant response range was from 2 to 10 and in- dividual scores represented the "true score" response expected from a respondent who used an additive combination of $X_{1}$ and $X_{2}$, who responded to the dependent response scale $\left(Y_{1}\right)$ with no random measurement error, and who was given a dependent response scale that permitted all responses to be made without any information loss.

The second dependent response scale $\left(Y_{2}\right)$ was created by summing $X_{1}$ and $X_{2}$ and then recoding the sum into five equal intervals (original $Y_{1}$ responses of 1 and 2 were recoded as 1 , responses of 3 and 4 were recoded as 2, and so forth) under the assumption of no measurement error. Hence, similar to the multiplicative example, $Y_{2}$ represented the response expected from a respondent who used an additive combination of $X_{1}$ and $X_{2}$, who responded to the dependent response scale $\left(Y_{2}\right)$ with no random measurement error, and who was given a dependent response scale that required information loss from the true $Y_{1}$ response. Table 3 contains all possible combinations of $X_{1}$ and $X_{2}$ variables with their associated $Y_{1}$ and $Y_{2}$ responses.

\section{Analysis and Results}

By definition, the regression of the additive combination of $X_{1}$ and $X_{2}$ onto $Y_{1}$ yields $R^{2}=1.00$. When the $Y_{2}$ dependent variable was regressed onto an additive combination of $X_{1}$ and $X_{2}, R^{2}=.951$. Hence, the information loss imposed on $Y_{2}$ would decrease the likelihood of detecting a true additive effect. Although Peters and Van Voorhis (1940) and Cohen (1983) demonstrated that the coarseness of the dependent scale will attenuate simple correlations, the same transformation will also attenuate additive main effects in multiple regression. Note that this type of result is also demonstrated in the $R_{\text {add }}^{2}$ column of Table 2 for the $Y_{2}$ dependent variable, whereas the opposite effect - that is, an inflation of $R_{\text {add }}^{2}$-occurred for $Y_{3}$ (however, dependent variables in Table 2 are transformations of a multiplicative model, not an additive model).

\section{Discussion}

The results clearly demonstrate that alternate 
Table 3

Complete Set of Independent Variables and Dependent Variables for Additive Example

\begin{tabular}{|c|c|c|c|}
\hline$X_{4}$ & $X_{2}$ & $Y_{\mathrm{i}}$ & $Y_{2}$ \\
\hline 1 & 1 & 2 & 1 \\
\hline 1 & 2 & 3 & 2 \\
\hline 1 & 3 & 4 & 2 \\
\hline 1 & 4 & 5 & 3 \\
\hline 1 & 5 & 6 & 3 \\
\hline 2 & 1 & 3 & 2 \\
\hline 2 & 2 & 4 & 2 \\
\hline 2 & 3 & 5 & 3 \\
\hline 2 & 4 & 6 & 3 \\
\hline 2 & 5 & 7 & 4 \\
\hline 3 & 1 & 4 & 2 \\
\hline 3 & 2 & 5 & 3 \\
\hline 3 & 3 & 6 & 3 \\
\hline 3 & 4 & 7 & 4 \\
\hline 3 & 5 & 8 & 4 \\
\hline 4 & 1 & 5 & 3 \\
\hline 4 & 2 & 6 & 3 \\
\hline 4 & 3 & 7 & 4 \\
\hline 4 & 4 & 8 & 4 \\
\hline 4 & 5 & 9 & 5 \\
\hline 5 & 1 & 6 & 3 \\
\hline 5 & 2 & 7 & 4 \\
\hline 5 & 3 & 8 & 4 \\
\hline 5 & 4 & 9 & 5 \\
\hline 5 & 5 & 10 & 5 \\
\hline
\end{tabular}

transformations of people's original responses can spuriously increase or decrease the expected effect size in moderated regression analysis. When a research design fails to provide a respondent with an overt response scale that is at least as large as their original response space, incorrect conclusions can be drawn from moderated regression analysis. This is a critical finding in the analysis of applied research in which the testing of interactive theories is frequent (e.g., Arnold, 1981; Prescott, 1986; Russell, 1985; Venkatraman, 1989). As noted earlier, Cronbach (1987) has called for alternative research strategies that are sensitive to interactive effects. A possible research strategy is the a priori identification of theoretical response domains before dependent response scales are developed. In turn, this identification process should help guarantee that the operational measurement of the dependent variable contains a sufficient number of response options.

Future research needs to examine the process underlying how information loss occurs and how characteristics of the measurement situation (e.g., anchor response format, predictor collinearity) influence the transformation. It is also possible that the size of the "reduction" task will determine the type of transformation used by the respondent. For example, if $X_{1}$ and $X_{2}$ are measured on 1- to 10-point scales, an additive model would yield a response space ranging from $Y=2$ to 20 whereas a multiplicative model would yield a response space ranging from 1 to 100 . Respondents may use different "reduction" processes to make small versus large dependent "response reductions."

The current examples implicitly assume some cognitive reduction process by the respondents; that is, the respondents have to somehow "squeeze" their latent dependent response to fit it onto the discrete overt response scale (Anderson, 1982, labeled this the "response function"). As one solution, researchers might consider using dependent scale formats that allow respondents to use a continuous response scale. Arnold's (1981) and Norman's (1986) use of a mark on a line segment permitted them to use the mark's distance from the end of the line (in millimeters) as a dependent scale value, effectively creating a continuous scale score. Although creating a somewhat cumbersome coding task, this procedure might permit more accurate reflection of a "true" underlying multiplicative response. With an increasing number of questionnaires being administered on personal computers, software that will present a relatively unobtrusive means of obtaining computer-generated response lines on the screen, with the respondent asked to use a mouse to place a mark on the line, might soon be seen. The computer would then generate a very precise measurement of the mark's position on the line, effectively eliminating the awkward requirements of using a ruler to quantify each person's response (the procedure has been used by 
Arnold, 1981, and Norman, 1986).

An additional approach to overcoming the cognitive problem in applied research of response reduction that is induced by scale coarseness would require investigators to shift their initial focus from the dependent variable to the independent variables. Specifically, investigators must identify the number of conceptually distinct levels possible for each independent variable before developing measurement scales for the dependent variable. For example, Arnold (1981) used the results of earlier work by Shanteau (1974) to identify five levels of expectancy. Under these circumstances, manipulation checks become doubly important to ensure that each independent variable has its intended effect and that the expected number of levels of effect are present. If manipulation of an independent variable yields more "levels" than anticipated, moderated regression results may be essentially uninterpretable.

Busemeyer and Jones (1983) noted that when respondents go through some unknown transformation between their true response and the observed response, interpretation of moderated regression analysis becomes problematic. The current study reaffirmed and extended this notion by showing how systematic response error is produced in the instance in which the number of scale options on $Y$ does not match the number of possible responses created by the multiplication of $X_{1}$ and $X_{2}$. The conflicting results of Arnold (1981) and Stahl and Harrell (1981), and potentially of any other empirical tests of interactive theories, may be due to the different number of dependent scale options used in each study.

As noted above, the current examples have all been depicted within a cognitively-oriented interaction model (Expectancy Theory). A multiplicative relationship was further assumed between fixed treatment effects (five "fixed" levels of $X_{1}$ and $X_{2}$ were specified a priori) and a dependent measure obtained by asking questions of the "treated" sample. These results should generalize to more complex models (e.g., conjunctive or disjunctive decision models), to random effects designs, and/or to archival measures as the dependent variable.

A more complex model would involve both additive and multiplicative effects, that is, $Y=X_{1}+X_{2}+X_{1} \times X_{2}$. Using this model, data were generated to create an initial $Y_{1}$ " "true" dependent response scale (with a maximum value of $5+5+5 \times 5=35$ ), and a $Y_{2}$ dependent response scale in which all values of $Y_{1}$ between 1 and 7 were coded as 1 , values between 8 and 14 were coded as 2, and so forth. For $Y_{1}$, $R_{\text {mult }}^{2}=1.00$ (by definition) and $R_{\text {add }}^{2}=.941$, yielding a difference of .059 . For $Y_{2}, R_{\mathrm{mult}}^{2}=.949$ and $R_{\text {add }}^{2}=.900$, yielding a difference of .049 . Hence, the same "grouping" transformation used for $Y_{2}$ throughout these examples resulted in an inflated interaction effect size for a "true" multiplicative model, and an attenuated interaction effect size for a "true" combined multiplicative-additive model. The same type of transformation will differentially impact the ability to detect alternate "true" models. Models with three- or four-way interaction terms and/or exponential functions will drastically increase the number of possible values in the dependent variable space. Accurate testing of these more elaborate functions would require a nearly continuous dependent response scale.

A similar situation arises when random effects designs are considered. If the independent variables are truly continuous and the levels observed by the investigator are specific to the sample at hand (e.g., the primary valence variable of Stahl \& Harrell, 1981), the investigator is again faced with an infinite dependent variable space and the need to use a continuous dependent response scale. Thus, for all but the simplest models, investigators should explore nontraditional means of operationalizing their dependent variables. These might include the cumbersome and labor intensive coding required to measure respondents' marks placed on an anchored line segment (Arnold, 1981), the development of optical scanning technology or software that accurately measures the distance down a line segment that respon- 
dents mark, or exploration of magnitude estimation procedures.

The examples throughout this paper focused on psychological dependent measures that require responses from people. Hence, it was implicitly assumed that respondents reduce or "squeeze" their "fine" latent dependent response to fit on a "coarse" dependent response scale. These arguments should also apply to studies using dependent variables "reduced" in some other manner. For example, Prescott (1986) examined the interaction effect of organizational environment and corporate strategy on an organization's return on investment (ROI) using archival data. In this instance, ROI was captured with a continuous measure in monetary terms. However, what if the archival data had been reduced to intervals or ranges of ROI values, a common condition in archival databases available to organizational psychologists? Although the term "response reduction" could be used to describe information loss due to coarse operationalizations of a fine dependent variable space, there are many ways in which a coarse operationalization can lead to information loss. Archivists making decisions of convenience to use coarse categories to summarize continuous data (e.g., as in $Y_{2}$ ) are but one example. Applied researchers must be alert to alternate sources of information loss if they are to accurately test all but the simplest models. Again, the interest is not in knowing a priori the optimal number of groupings to minimize information loss (Cox, 1950) or in identifying the effect that all possible transformations might have on the ability to detect nonlinear models. Rather, the goal is to prevent spurious conclusions from being drawn when research designs unintentionally lose critical information from the dependent variable, through response reduction or any other means.

\section{Related Measurement Issues}

As noted above, it could be argued that in survey research the measurement of the dependent variable becomes continuous when people's responses are summed across multiple dependent scale items. For example, 10 dependent scale items with 1- to 5-point response scales are typically summed to yield a dependent scale score ranging from 10 to 50 . Unfortunately, although an investigator may sum responses across items to yield a composite dependent scale, responses are made sequentially to each item. Adding item responses to form a dependent scale score effectively controls for the effects of random influences on people's responses. However, it does not necessarily negate any information loss or "reduction" that might have occurred in their item responses prior to summation into a single scale score. In fact, the systematic error may cumulate across the sum. Again, more basic research is needed concerning the response reduction process.

Finally, it should also be noted that the effect of information loss described here is different from simple range restriction. Range restriction occurs when a measure contains observations from only a portion of the potential range of responses. Correlations between a measure suffering from range restriction and any predictor variable will then be attenuated. In contrast, the information loss due to "response reduction" does not eliminate observations from a portion of the potential range of responses. The response reduction involves the respondent's placement of all potential responses on a scale that does not have enough options to differentiate among all of the responses. The variance is not necessarily reduced; rather, the number of options within a given range is limited. The results here indicate that, depending on how people "reduce" their responses, the result could yield a spurious attenuation or increase in effect size.

The analyses presented here demonstrate that the number of response options on the dependent scale can severely influence the effect sizes in moderated regression analysis. As demonstrated above, it is critical to give respondents enough space when searching for moderator effects in applied psychological research and/or to be alert for sources of information loss in archival data. More basic research is needed regarding (1) how peo- 
ple actually reduce "true" continuous responses to yield an overt response to discrete response options and (2) the relative efficacy of using continuous response scales to operationalize dependent responses.

\section{References}

Anderson, N. H. (1982). Methods of information integration theory. New York: Academic Press.

Arnold, H. J. (1981). A test of the multiplicative hypothesis of expectancy-valence theories of work motivation. Academy of Management Journal, 24, 128-141.

Birnbaum, M., \& Veit, C. T. (1974). Scale convergence as a criterion for rescaling: Information integration with difference, ratio, and averaging tasks. Perception and Psychophysics, 15, 7-15.

Bobko, P. (1986). A solution to some dilemmas when testing hypothesis about ordinal interactions. Journal of Applied Psychology, 77, 323-326.

Busemeyer, J. R., \& Jones, L. E. (1983). Analysis of multiplicative combination rules when the causal variables are measured with error. Psychological Bulletin, 93, 549-562.

Chow, G. (1960). Test of equality between sets of coefficients in two linear regressions. Econometrica, 28, 591-604.

Cicchetti, D. V., Showalter, D., \& Tyrer, P. J. (1985). The effect of number of rating scale categories on levels of interrater reliability: A monte carlo investigation. Applied Psychological Measurement, 9, 31-36.

Cohen, J. (1983). The cost of dichotomization. Applied Psychological Measurement, 7, 249-253.

Cohen, J., \& Cohen, P. (1983). Applied multiple regression/correlation analysis for the behavioral sciences (2nd ed.). Hillsdale NJ: Erlbaum.

Cox, D. R. (1950). Note on grouping. Journal of the American Statistical Association, 52, 543-547.

Cronbach, L. J. (1987). Statistical tests for moderator variables: Flaws in analyses recently proposed. Psychological Bulletin, 102, 414-417.

Drazin, R., \& Van Der Ven, A. H. (1985). An examination of alternative forms of fit in contingency theory. Administrative Science Quarterly, 30, 514-539.

Edwards, A. L., \& Kenney, K. C. (1946). A comparison of the Thurstone and Likert techniques of attitude scale construction. Journal of Applied Psychology, 30, $72-83$.

Jenkins, G. D., \& Taber, T. D. (1977). A monte carlo study of factors affecting three indices of composite scale reliability. Journal of Applied Psychology, 62, 392-398.
Likert, R. A. (1932). A technique for the measurement of attitudes. Archives of Psychology, New York, No. 140.

Morris, J. H., Sherman, J. D., \& Mansfield, E. R. (1986). Failures to detect moderating effects with ordinary least squares-moderated multiple regression: Some reasons and a remedy. Psychological Bulletin, 99, 282-288.

Norman, K. L. (1986). Importance of factors in the review of grant proposals. Journal of Applied Psychology, 71, 156-162.

Paunonen, S. V., \& Jackson, D. N. (1988). Type I error rates for moderated multiple regression analysis. Journal of Applied Psychology, 73, 569-573.

Peters, C. C., \& Van Voorhis, W. R. (1940). Statistical procedures and their mathematical bases. New York: McGraw-Hill.

Prescott, J. E. (1986). Environment as a moderator of strategy-performance relationship. Academy of Management Journal, 29, 329-346.

Rasmussen, J. L. (1989). Analysis of Likert-scale data: A reinterpretation of Gregoire and Driver. Psychological Bulletin, 105, 167-170.

Russell, C. J. (1985). Individual decision processes in an assessment center. Journal of Applied Psychology, $70,737-746$.

Saunders, D. R. (1955). The "moderator variable" as a useful tool in prediction. Proceedings of the 1954 Invitational Conference on Testing Problems (pp. 54-58). Princeton NJ: Educational Testing Service.

Saunders, D. R. (1956). Moderator variables in prediction. Educational and Psychological Measurement, 16, 209-222.

Schwab, D. P. (1980). Construct validity in organizational behavior. In B. M. Staw \& L. L. Cummings (Eds.), Research in organizational behavior (pp. 3-43). Greenwich CT: JAI Press.

Shanteau, J. C. (1974). Component processes in risky decision making. Journal of Experimental Psychology, 103, 680-691.

Sockloff, A. (1976a). The analysis of nonlinearity via linear regression with polynomial and product variables: An examination. Review of Educational Research, 46, 267-291.

Sockloff, A. (1976b). Spurious product correlation. Educational and Psychological Measurement, 36, 33-44.

Stahl, M. J., \& Harrell, A. M. (1981). Modeling effort decisions with behavioral decision theory: Toward an individual differences model of expectancy theory. Organizational Behavior and Human Performance, 27, 303-325.

Venkatraman, N. (1989). The concept of fit in strategy research: Toward verbal and statistical correspondence. Academy of Management Review, 14, 423-444. 
Zedeck, S. (1971). Problems with the use of "moderator" variables. Psychological Bulletin, 76, 295-310.

\section{Acknowledgments}

The authors thank Edward Alf, Jerome Busemeyer, Martin Evans, Frank Schmidt, and Eugene Stone for their comments on an earlier draft of this report. Partial support for this project was provided by the Coordinating Council for Business Studies Research Grants program of Rutgers University. Revisions were made while the senior author held a visiting appointment at the Krannert Graduate School of Management, Purdue University. The authors also thank an anonymous reviewer for suggesting questions of generalization to more complex models.

\section{Author's Address}

Send requests for reprints or further information to Craig J. Russell, Department of Management, College of Business, Louisiana State University, Baton Rouge LA 70803-6312, U.S.A. 